\title{
Explicit criteria for the oscillation of second-order differential equations with several sub-linear neutral coefficients
}

\author{
Shyam Sundar Santra ${ }^{1}$, Tanusri Ghosh ${ }^{1}$ and Omar Bazighifan ${ }^{2,3^{*}}$ (I)
}

\section{"Correspondence:}

o.bazighifan@gmail.com

${ }^{2}$ Department of Mathematics,

Faculty of Science, Hadhramout

University, Hadhramout 50512, Yemen

${ }^{3}$ Department of Mathematics, Faculty of Education, Seiyun University, Hadhramout 50512, Yemen

Full list of author information is available at the end of the article

\begin{abstract}
In this work, we present sufficient conditions for oscillation of all solutions of a second-order functional differential equation. We consider two special cases when $\gamma>\beta$ and $\gamma<\beta$. This new theorem complements and improves a number of results reported in the literature. Finally, we provide examples illustrating our results and state an open problem.
\end{abstract}

MSC: $34 \mathrm{C} 10 ; 34 \mathrm{~K} 11$

Keywords: Oscillation; Non-oscillation; Neutral; Second-order

\section{Introduction}

Delay differential equations are widely used in mathematical modeling to describe physical and biological systems, often inducing oscillatory behavior $[1-4,8,13,14,17,18,24-$ 26, 28-35].

In the literature, numerous mathematical models with different levels of complexity have been proposed for delay differential equations in order to represent the cardiovascular system (CVS).

The pioneering and remarkable paper of Ottesen [27] shows how to use delay differential equations to solve a cardiovascular model that has a discontinuous derivative. Ottesen [27] also illustrated that complex dynamic interactions between nonlinear behaviors and delays associated with the autonomic-cardiac regulation may cause instability [5].

Moreover, a model-based approach to stability analysis of autonomic-cardiac regulation was studied in [5]; specifically, it is important to underline that the autonomic-cardiac regulation operates by the interaction between autonomic nervous system (ANS) and cardiovascular system (CVS) [5].

It is clear that mathematical analysis based on physics-based models can be a versatile tool in examining delay differential equations from the point of view of biological systems.

In this article we consider the neutral differential equation

$$
\left(r(t)\left(w^{\prime}(t)\right)^{\gamma}\right)^{\prime}+q(t) x^{\beta}(\vartheta(t))=0, \quad t \geq t_{0},
$$

(c) The Author(s) 2020. This article is licensed under a Creative Commons Attribution 4.0 International License, which permits use, sharing, adaptation, distribution and reproduction in any medium or format, as long as you give appropriate credit to the original author(s) and the source, provide a link to the Creative Commons licence, and indicate if changes were made. The images or other third party material in this article are included in the article's Creative Commons licence, unless indicated otherwise in a credit line to the material. If material is not included in the article's Creative Commons licence and your intended use is not permitted by statutory regulation or exceeds the permitted use, you will need to obtain permission directly from the copyright holder. To view a copy of this licence, visit http://creativecommons.org/licenses/by/4.0/. 
where $w(t)=x(t)+\sum_{i=1}^{m} p_{i}(t) x^{\alpha_{i}}\left(\varsigma_{i}(t)\right), \alpha_{i}$ for $i=1,2, \ldots, m, \gamma$ and $\beta$ are the quotients of odd positive integers. Throughout this work, we suppose that:

(A1) $\vartheta, \varsigma_{i} \in C\left(\left[t_{0}, \infty\right), \mathbb{R}_{+}\right), \varsigma_{i} \in C^{2}\left(\left[t_{0}, \infty\right), \mathbb{R}_{+}\right), \vartheta(t)<t, \varsigma_{i}(t)<t, \lim _{t \rightarrow \infty} \vartheta(t)=\infty$, $\lim _{t \rightarrow \infty} S_{i}(t)=\infty$ for all $i=1,2, \ldots$.

(A2) $r \in C^{1}\left(\left[t_{0}, \infty\right), \mathbb{R}_{+}\right), q \in C\left(\left[t_{0}, \infty\right), \mathbb{R}_{+}\right) ; 0 \leq q(t)$ for all $t \geq 0 ; q(t)$ is not identically zero in any interval $[b, \infty)$.

(A3) $\lim _{t \rightarrow \infty} R(t)=\infty$, where $R(t)=\int_{t_{1}}^{t} r^{-1 / \gamma}(s) d s$.

(A4) $p_{i}:\left[t_{0}, \infty\right) \rightarrow \mathbb{R}^{+}$are continuous functions for $i=1,2, \ldots, m$.

In 1978, Brands [11] proved that, for each bounded delay $\vartheta(t)$, the equation

$$
x^{\prime \prime}(t)+q(t) x(t-\vartheta(t))=0
$$

is oscillatory if and only if the equation

$$
x^{\prime \prime}(t)+q(t) x(t)=0
$$

is oscillatory. In $[12,15]$ Chatzarakis et al. considered a more general equation

$$
\left(r\left(x^{\prime}\right)^{\beta}\right)^{\prime}(t)+q(t) x^{\beta}(\vartheta(t))=0,
$$

and established new oscillation criteria for (2) when $\lim _{t \rightarrow \infty} R(t)=\infty$ and $\lim _{t \rightarrow \infty} R(t)<$ $\infty$.

Wong [37] obtained the oscillation conditions of

$$
(x(t)+p x(t-\varsigma))^{\prime \prime}+q(t) f(x(t-\vartheta))=0, \quad-1<p<0,
$$

in which the neutral coefficient and delays are constants. However, we have seen in $[6,16]$ that the authors Baculikovǎ and Džurina studied

$$
\left(r(t)\left(w^{\prime}(t)\right)^{\gamma}\right)^{\prime}+q(t) x^{\beta}(\vartheta(t))=0, \quad w(t)=x(t)+p(t) x(\varsigma(t)), \quad t \geq t_{0},
$$

and established the oscillation of solutions of (3) using comparison techniques when $\gamma=\beta=1,0 \leq p(t)<\infty$ and $\lim _{t \rightarrow \infty} R(t)=\infty$. In the same technique, Baculikova and Džurina [7] considered (3) and obtained oscillation conditions of (3) by considering the assumptions $0 \leq p(t)<\infty$ and $\lim _{t \rightarrow \infty} R(t)=\infty$. In [36], Tripathy et al. studied (3) and established several conditions of the solutions of (3) by considering the assumptions $\lim _{t \rightarrow \infty} R(t)=\infty$ and $\lim _{t \rightarrow \infty} R(t)<\infty$ for different ranges of the neutral coefficient $p$. In [9], Bohner et al. obtained sufficient conditions for oscillation of solutions of (3) when $\gamma=\beta, \lim _{t \rightarrow \infty} R(t)<\infty$, and $0 \leq p(t)<1$. Grace et al. [19] studied the oscillation of (3) when $\gamma=\beta$ and by considering the assumptions $\lim _{t \rightarrow \infty} R(t)<\infty, \lim _{t \rightarrow \infty} R(t)=\infty$, and $0 \leq p(t)<1$. In [22], Li et al. established sufficient conditions for the oscillation of the solutions of (3) under the assumptions $\lim _{t \rightarrow \infty} R(t)<\infty$ and $p(t) \geq 0$. Karpuz and Santra [21] considered the equation

$$
\left(r(t)(x(t)+p(t) x(\varsigma(t)))^{\prime}\right)^{\prime}+q(t) f(x(\vartheta(t)))=0
$$


by considering the assumptions $\lim _{t \rightarrow \infty} R(t)<\infty$ and $\lim _{t \rightarrow \infty} R(t)=\infty$ for different ranges of $p$.

In fact, equation (1) (that is, half-linear/Emden-Fowler differential equation) arises in a variety of real world problems such as in the study of non-Newtonian fluid theory, the turbulent flow of a polytrophic gas in a porous medium [10, 23]. Neutral differential equations have several applications in the natural sciences and engineering. For example, they often appear in models biological (see, e.g., [20]). In this paper, we restrict our attention to studying oscillation and non-oscillation of (1).

\section{Preliminary results}

To simplify our notation, for any function $\rho:\left[t_{0}, \infty\right) \rightarrow \mathbb{R}^{+}$which is positive, continuous decreasing to zero, we set

$$
\begin{aligned}
& P(t)=\left(1-\sum_{i=1}^{m} \alpha_{i} p_{i}(t)-\frac{1}{\rho(t)} \sum_{i=1}^{m}\left(1-\alpha_{i}\right) p_{i}(t)\right) \geq 0 ; \\
& Q_{1}(t)=q(t) P^{\beta}(\vartheta(t)) ; \\
& Q_{2}(t)=q(t) P^{\beta}(\vartheta(t)) \rho^{\beta-1}(\vartheta(t)) ; \\
& Q_{3}(t)=q(t) P^{\beta}(\vartheta(t)) R^{\beta-1}(\vartheta(t)) ; \\
& Q_{4}(t)=q(t) P^{\beta}(\vartheta(t)) R^{\beta}(\vartheta(t)) ; \\
& U(t)=\int_{t}^{\infty} q(\zeta) x^{\beta}(\vartheta(\zeta)) d \zeta \geq 0 .
\end{aligned}
$$

We need the following lemmas for our work in the sequel.

Lemma 2.1 ([20]) If $a$ and $b$ are nonnegative, then

$$
a^{\alpha} b^{1-\alpha} \leq \alpha a+(1-\alpha) b \text { for } 0<\alpha \leq 1,
$$

where equality holds if and only if $a=b$.

Lemma 2.2 Let (A1)-(A4) hold for $t \geq t_{0}$. If $x$ is an eventually positive solution of (1), then w satisfies

$$
w(t)>0, \quad w^{\prime}(t)>0, \quad \text { and } \quad\left(r\left(w^{\prime}\right)^{\gamma}\right)^{\prime}(t) \leq 0 \quad \text { for } t \geq t_{1} .
$$

Proof Let $x$ be an eventually positive solution of (1). Hence, $w(t)>0$, and there exists $t_{0} \geq 0$ such that $x(t)>0, x\left(s_{i}(t)\right)>0$, and $x(\vartheta(t))>0$ for all $t \geq t_{0}$ and for all $i=1,2, \ldots$. From (1) it follows that

$$
\left(r(t)\left(w^{\prime}(t)\right)^{\gamma}\right)^{\prime}=-q(t) x^{\beta}(\vartheta(t)) \leq 0 \quad \text { for } t \geq t_{0} .
$$

Therefore, $r(t)\left(w^{\prime}(t)\right)^{\gamma}$ is nonincreasing for $t \geq t_{0}$. Assume that $r(t)\left(w^{\prime}(t)\right)^{\gamma}<0$ for $t \geq t_{1}>$ $t_{0}$. Hence,

$$
r(t)\left(w^{\prime}(t)\right)^{\gamma} \leq r\left(t_{1}\right)\left(w^{\prime}\left(t_{1}\right)\right)^{\gamma}<0 \text { for all } t \geq t_{1},
$$


that is,

$$
w^{\prime}(t) \leq\left(\frac{r\left(t_{1}\right)}{r(t)}\right)^{1 / \gamma} w^{\prime}\left(t_{1}\right) \quad \text { for } t \geq t_{1}
$$

Using integration from $t_{1}$ to $t$, we have

$$
w(t) \leq w\left(t_{1}\right)+\left(r\left(t_{1}\right)\right)^{1 / \gamma} w^{\prime}\left(t_{1}\right) R(t) \rightarrow-\infty
$$

as $t \rightarrow \infty$ due to (A3), which is a contradiction to $w(t)>0$.

Therefore $r(t)\left(w^{\prime}(t)\right)^{\gamma}>0$ for all $t \geq t_{1}$. From $r(t)\left(w^{\prime}(t)\right)^{\gamma}>0$ and $r(t)>0$, it follows that $w^{\prime}(t)>0$. This completes the proof.

Lemma 2.3 Let (A1)-(A4) hold for $t \geq t_{0}$. If $x$ is an eventually positive solution of (1), then w satisfies

$$
w(t) \geq(r(t))^{1 / \gamma} w^{\prime}(t) R(t) \quad \text { for } t \geq t_{1}
$$

and

$$
\frac{w(t)}{R(t)} \text { is decreasing for } t \geq t_{1}
$$

Proof Proceeding as in the proof of Lemma 2.2, we obtain (4) for $t \geq t_{1}$. Since $r(t)\left(w^{\prime}(t)\right)^{\gamma}$ is decreasing, we have

$$
\begin{aligned}
w(t) & \geq \int_{t_{1}}^{t}(r(\eta))^{1 / \gamma} w^{\prime}(\eta) \frac{1}{(r(\eta))^{1 / \gamma}} d \eta \\
& \geq(r(t))^{1 / \gamma} w^{\prime}(t) \int_{t_{1}}^{t} \frac{1}{(r(\eta))^{1 / \gamma}} d \eta \\
& \geq(r(t))^{1 / \gamma} w^{\prime}(t) R(t) .
\end{aligned}
$$

Again, using the previous inequality, we have

$$
\left(\frac{w(t)}{R(t)}\right)^{\prime}=\frac{(r(t))^{1 / \gamma} w^{\prime}(t) R(t)-w(t)}{(r(t))^{1 / \gamma} R^{2}(t)} \leq 0
$$

We conclude that $\frac{w(t)}{R(t)}$ is decreasing for $t \geq t_{1}$. This completes the proof.

Lemma 2.4 Let (A1)-(A4) hold for $t \geq t_{0}$. If $x$ is an eventually positive solution of (1), then w satisfies

$$
x(t) \geq P(t) w(t) \quad \text { for } t \geq t_{1} .
$$


Proof Let $x$ be an eventually positive solution of (1). Hence, $w(t)>0$, and there exists $t_{0} \geq 0$ such that

$$
\begin{aligned}
x(t) & =w(t)-\sum_{i=1}^{m} p_{i}(t) x^{\alpha_{i}}\left(\varsigma_{i}(t)\right) \\
& \geq w(t)-\sum_{i=1}^{m} p_{i}(t) w^{\alpha_{i}}\left(\varsigma_{i}(t)\right) \\
& \geq w(t)-\sum_{i=1}^{m} p_{i}(t) w^{\alpha_{i}}(t) \\
& \geq w(t)-\sum_{i=1}^{m} p_{i}(t)\left(\alpha_{i} w(t)-\left(1-\alpha_{i}\right)\right) \\
& =\left(1-\sum_{i=1}^{m} \alpha_{i} p_{i}(t)\right) w(t)-\sum_{i=1}^{m}\left(1-\alpha_{i}\right) p_{i}(t)
\end{aligned}
$$

using Lemma 2.1. Since $w(t)$ is positive and increasing and $\rho(t)$ is positive and decreasing to zero, there is $t_{0} \geq t_{1}$ such that

$$
w(t) \geq \rho(t) \quad \text { for } t \geq t_{1} .
$$

Using (7) in (6), we obtain

$$
x(t) \geq P(t) w(t) .
$$

This completes the proof.

Lemma 2.5 Let (A1)-(A4) hold for $t \geq t_{0}$. If $x$ is an eventually positive solution of (1), then there exist $t_{1}>t_{0}$ and $\delta>0$ such that

$$
\begin{aligned}
& 0<w(t) \leq \delta R(t) \text { and } \\
& R(t)\left[\int_{t}^{\infty} q(\zeta) x^{\beta}(\vartheta(\zeta)) d \zeta\right]^{1 / \gamma} \leq w(t)
\end{aligned}
$$

hold for all $t \geq t_{1}$.

Proof Let $x$ be an eventually positive solution of (1). Then there exists $t_{0}>0$ such that $x(t)>0, x\left(\varsigma_{i}(t)\right)>0$, and $x(\vartheta(t))>0$ for all $t \geq t_{0}$ and for all $i=1,2, \ldots$. So, there exists $t_{1}>t_{0}$ such that Lemma 2.2 holds true and $w$ satisfies (4) for $t \geq t_{1}$. From $r(t)\left(w^{\prime}(t)\right)^{\gamma}>0$ and being nonincreasing, we have

$$
w^{\prime}(t) \leq\left(\frac{r\left(t_{1}\right)}{r(t)}\right)^{1 / \gamma} w^{\prime}\left(t_{1}\right) \quad \text { for } t \geq t_{1} .
$$

Integrating this inequality from $t_{1}$ to $t$,

$$
w(t) \leq w\left(t_{1}\right)+\left(r\left(t_{1}\right)\right)^{1 / \gamma} w^{\prime}\left(t_{1}\right) R(t) .
$$


Since $\lim _{t \rightarrow \infty} R(t)=\infty$, there exists a positive constant $\delta$ such that (8) holds. On the other hand, $\lim _{t \rightarrow \infty} r(t)\left(w^{\prime}(t)\right)^{\gamma}$ exists, and integrating (1) from $t$ to $a$, we obtain

$$
r(a)\left(w^{\prime}(a)\right)^{\gamma}-r(t)\left(w^{\prime}(t)\right)^{\gamma}=-\int_{t}^{a} q(\eta) x^{\beta}(\vartheta(\eta)) d \eta .
$$

Taking limit as $a \rightarrow \infty$,

$$
r(t)\left(w^{\prime}(t)\right)^{\gamma} \geq \int_{t}^{\infty} q(\eta) x^{\beta}(\vartheta(\eta)) d \eta
$$

that is,

$$
w^{\prime}(t) \geq\left[\frac{1}{r(t)} \int_{t}^{\infty} q(\eta) x^{\beta}(\vartheta(\eta)) d \eta\right]^{1 / \gamma} .
$$

Therefore,

$$
\begin{aligned}
w(t) & \geq \int_{t_{1}}^{t}\left[\frac{1}{r(\eta)} \int_{\eta}^{\infty} q(s) x^{\beta}(\vartheta(s)) d s\right]^{1 / \gamma} d \eta \\
& \geq \int_{t_{1}}^{t}\left[\frac{1}{r(\eta)} \int_{t}^{\infty} q(s) x^{\beta}(\vartheta(s)) d s\right]^{1 / \gamma} d \eta \\
& =R(t)\left[\int_{t}^{\infty} q(s) x^{\beta}(\vartheta(s)) d s\right]^{1 / \gamma} .
\end{aligned}
$$

This completes the proof.

\section{Sufficient conditions for oscillations}

Theorem 3.1 Let (A1)-(A4) hold for $t \geq t_{0}$. If

(A5) $\int_{0}^{\infty} Q_{1}(\eta) d \eta=\infty$

holds, then every solution of (1) is oscillatory.

Proof Let $x$ be an eventually positive solution of (1). Then there exists $t_{0}>0$ such that $x(t)>0, x\left(\varsigma_{i}(t)\right)>0$, and $x(\vartheta(t))>0$ for all $t \geq t_{0}$ and for all $i=1,2, \ldots$. Applying Lemmas 2.2 and 2.4 for $t \geq t_{1}>t_{0}$, we conclude that $w$ satisfies (4), $w$ is increasing, and $x(t) \geq$ $P(t) w(t)$ for all $t \geq t_{1}$. From (1), we have

$$
\left(r(t)\left(w^{\prime}(t)\right)^{\gamma}\right)^{\prime}+q(t) P^{\beta}(\vartheta(t)) w^{\beta}(\vartheta(t)) \leq 0
$$

for $t \geq t_{1}$. Applying (4), we conclude that $\lim _{t \rightarrow \infty}\left(r(t)\left(w^{\prime}(t)\right)^{\gamma}\right)$ exists, and there exist $t_{2}>t_{1}$ and a number $c>0$ such that $w(t) \geq c$ for $t \geq t_{2}$. Integrating (11) from $t_{2}$ to $t$, we have

$$
c^{\beta} \int_{t_{2}}^{t} q(s) P^{\beta}(\vartheta(s)) d s \leq-\left[r(s)\left(w^{\prime}(s)\right)^{\gamma}\right]_{t_{2}}^{t}<\infty \quad \text { as } t \rightarrow \infty
$$

which is a contradiction to (A5).

The case where $x$ is an eventually negative solution is similar, and we omit it here. Thus, the proof is complete. 
Remark 3.1 Theorem 3.1 holds for any $\beta$ and $\gamma$.

Next, we obtain an oscillation result for equation (1) in the case $\beta>1$.

Theorem 3.2 Let (A1)-(A4) hold for $t \geq t_{0}$. If

(A6) $\int_{0}^{\infty} Q_{2}(\eta) d \eta=\infty$

holds, then every solution of (1) is oscillatory.

Proof Proceeding as in the proof of Theorem 3.1, we obtain (11). Applying (7) in (11), we have

$$
\left(r(t)\left(w^{\prime}(t)\right)^{\gamma}\right)^{\prime}+q(t) P^{\beta}(\vartheta(t)) \rho^{\beta-1}(\vartheta(t)) w(\vartheta(t)) \leq 0 .
$$

The rest of the proof is similar to that of Theorem 3.1, and hence the details are omitted.

Next, we obtain an oscillation result for equation (1) in the case $0<\beta<1$.

Theorem 3.3 Let (A1)-(A4) hold for $t \geq t_{0}$. If

(A7) $\int_{0}^{\infty} Q_{3}(\eta) d \eta=\infty$

holds, then every solution of (1) is oscillatory.

Proof Proceeding as in the proof of Theorem 3.1 we obtain (11). Now (11) can be written as

$$
\left(r(t)\left(w^{\prime}(t)\right)^{\gamma}\right)^{\prime}+q(t) P^{\beta}(\vartheta(t)) R^{\beta-1}(\vartheta(t)) \frac{w^{\beta-1}(\vartheta(t))}{R^{\beta-1}(\vartheta(t))} w(\vartheta(t)) \leq 0
$$

for $t \geq t_{2}>t_{1}$. Since $\frac{w(t)}{R(t)}$ is decreasing, there is a constant $k$ such that

$$
\frac{w(t)}{R(t)} \leq k \quad \text { for } t \geq t_{2}
$$

Using (14) and $\beta<1$ in (13), we have

$$
\left(r(t)\left(w^{\prime}(t)\right)^{\gamma}\right)^{\prime}+q(t) \frac{P^{\beta}(\vartheta(t)) R^{\beta-1}(\vartheta(t))}{k^{1-\beta}} w(\vartheta(t)) \leq 0 .
$$

The rest of the proof is similar to that of Theorem 3.2, and hence it is omitted.

Next, we assume that there exists a constant $\beta_{1}$, the quotient of odd positive integers such that $0<\beta<\beta_{1}<\gamma$.

Theorem 3.4 Let (A1)-(A4) hold for $t \geq t_{0}$. If

$$
\text { (A8) } \int_{0}^{\infty} Q_{4}(\eta) d \eta=\infty
$$

holds, then every solution of (1) is oscillatory.

Proof Let $x$ be an eventually positive solution of (1). So, there exists $t_{0}>0$ such that $x(t)>$ $0, x\left(s_{i}(t)\right)>0$, and $x(\vartheta(t))>0$ for all $t \geq t_{0}$ and for all $i=1,2, \ldots$ Applying Lemmas 2.2 and 
2.5 for $t \geq t_{1}>t_{0}$, we conclude that $w$ satisfies (4), (8), and (9) for all $t \geq t_{1}$. We can find $t_{1}>0$ such that

$$
w(t) \geq R(t) U^{1 / \gamma}(t) \geq 0 \quad \text { for } t \geq t_{1} .
$$

Using (5), (8), $\beta-\beta_{1}<0$, and (15), we have

$$
\begin{aligned}
x^{\beta}(t) & \geq P^{\beta}(t) w^{\beta-\beta_{1}}(t) w^{\beta_{1}}(t) \geq P^{\beta}(t)(\delta R(t))^{\beta-\beta_{1}} w^{\beta_{1}}(t) \\
& \geq P^{\beta}(t)(\delta R(t))^{\beta-\beta_{1}}\left(R(t) U^{1 / \gamma}(t)\right)^{\beta_{1}}=P^{\beta}(t) \delta^{\beta-\beta_{1}} R^{\beta}(t) U^{\beta_{1} / \gamma}(t) \quad \text { for } t \geq t_{2} .
\end{aligned}
$$

Since $U^{\prime}(t)=-q(t) x^{\beta}(\vartheta(t)) \leq 0, t \geq t_{2}$, that is, $w$ is nonincreasing, then the last inequality becomes

$$
\begin{aligned}
x^{\beta}(\vartheta(\eta)) & \geq P^{\beta}(\vartheta(\eta)) \delta^{\beta-\beta_{1}} R^{\beta}(\vartheta(\eta)) U^{\beta_{1} / \gamma}(\vartheta(\eta)) \\
& \geq P^{\beta}(\vartheta(\eta)) \delta^{\beta-\beta_{1}} R^{\beta}(\vartheta(\eta)) U^{\beta_{1} / \gamma}(\eta) .
\end{aligned}
$$

Therefore,

$$
\left(U^{1-\beta_{1} / \gamma}(t)\right)^{\prime}=\left(1-\frac{\beta_{1}}{\gamma}\right) w^{-\beta_{1} / \gamma}(t) U^{\prime}(t)
$$

Integrating (17) from $t_{2}$ to $t$ and then using the fact that $U>0$, we find

$$
\begin{aligned}
\infty & >U^{1-\beta_{1} / \gamma}\left(t_{2}\right) \geq\left(1-\frac{\beta_{1}}{\gamma}\right)\left[-\int_{t_{2}}^{t} U^{-\beta_{1} / \gamma}(\eta) U^{\prime}(\eta) d \eta\right] \\
& =\left(1-\frac{\beta_{1}}{\gamma}\right)\left[\int_{t_{2}}^{t} U^{-\beta_{1} / \gamma}(\eta)\left(q(\eta) x^{\beta}(\vartheta(\eta))\right) d \eta\right] \\
& \geq \frac{\left(1-\frac{\beta_{1}}{\gamma}\right)}{\delta^{\left(\beta_{1}-\beta\right)}}\left[\int_{t_{2}}^{t} q(\eta) P^{\beta}(\vartheta(\eta)) R^{\beta}(\vartheta(\eta)) d \eta\right],
\end{aligned}
$$

which contradicts (A8) as $t \rightarrow \infty$.

This completes the proof.

Next, we assume that there exists a constant $\beta_{2}$, the quotient of odd positive integers such that $\gamma<\beta_{2}<\beta$.

Theorem 3.5 Let (A1)-(A4) hold for $t \geq t_{0}, \vartheta^{\prime}(t) \geq \vartheta_{0}>0$ and $r(t)$ is nondecreasing. If

$$
\text { (A9) } \int_{0}^{\infty}\left[\frac{1}{r(\eta)} \int_{\eta}^{\infty} Q_{1}(\zeta) d \zeta\right]^{1 / \gamma} d \eta=\infty
$$

holds, then every solution of (1) is oscillatory.

Proof Let $x$ be an eventually positive solution of (1). Then there exists $t_{0}>0$ such that $x(t)>0, x\left(s_{i}(t)\right)>0$, and $x(\vartheta(t))>0$ for all $t \geq t_{0}$ and $i=1,2, \ldots$ Applying Lemmas 2.2 and 2.4 for $t \geq t_{1}>t_{0}$, we conclude that $w$ satisfies (4), $w$ is increasing, and $x(t) \geq P(t) w(t)$ for all $t \geq t_{1}$. So,

$$
x^{\beta}(t) \geq P^{\beta}(t) w^{\beta}(t) \geq P^{\beta}(t) w^{\beta-\beta_{2}}(t) w^{\beta_{2}}(t) \geq P^{\beta}(t) w^{\beta-\beta_{2}}\left(t_{1}\right) w^{\beta_{2}}(t)
$$


implies that

$$
x^{\beta}(\vartheta(t)) \geq P^{\beta}(\vartheta(t)) w^{\beta-\beta_{2}}\left(t_{1}\right) w^{\beta_{2}}(\vartheta(t)) \text { for } t \geq t_{2}>t_{1} .
$$

Using (10) and (18), we have

$$
r(t)\left(w^{\prime}(t)\right)^{\gamma} \geq w^{\beta-\beta_{2}}\left(t_{1}\right)\left[\int_{t}^{\infty} q(\eta) P^{\beta}(\vartheta(\eta)) d \eta\right] w^{\beta_{2}}(\vartheta(t))
$$

for $t \geq t_{2}$. From $r(t)\left(w^{\prime}(t)\right)^{\gamma}$ being nonincreasing and $\vartheta(t) \leq t$, we have

$$
r(\vartheta(t))\left(w^{\prime}(\vartheta(t))\right)^{\gamma} \geq r(t)\left(w^{\prime}(t)\right)^{\gamma} .
$$

Using the last inequality in (19) and then dividing by $r(\vartheta(t)) w^{\beta_{2}}(\vartheta(t))>0$, and then operating the power $1 / \gamma$ on both sides, we get

$$
\frac{w^{\prime}(\vartheta(t))}{w^{\beta_{2} / \gamma}(\vartheta(t))} \geq\left[\frac{w^{\beta-\beta_{2}}\left(t_{1}\right)}{r\left(\vartheta_{0}(t)\right)} \int_{t}^{\infty} q(\eta) P^{\beta}(\vartheta(\eta)) d \eta\right]^{1 / \gamma}
$$

for $t \geq t_{2}$. Multiplying the left-hand side by $\vartheta^{\prime}(t) / \vartheta_{0} \geq 1$ and integrating from $t_{2}$ to $t$, we find

$$
\begin{aligned}
& \frac{1}{\vartheta_{0}} \int_{t_{2}}^{t} \frac{w^{\prime}(\vartheta(\eta)) \vartheta^{\prime}(\eta)}{w^{\beta_{2} / \gamma}(\vartheta(\eta))} d \eta \\
& \quad \geq w^{\left(\beta-\beta_{2}\right) / \gamma}\left(t_{1}\right) \int_{t_{2}}^{t}\left[\frac{1}{r(\vartheta(\eta))} \int_{\eta}^{\infty} q(\zeta) P^{\beta}(\vartheta(\zeta)) d \zeta\right]^{1 / \gamma} d \eta t \geq t_{2}
\end{aligned}
$$

Since $\gamma<\beta_{2}, r(\vartheta(\eta)) \leq r(\eta)$ and

$$
\frac{1}{\vartheta_{0}\left(1-\beta_{2} / \gamma\right)}\left[w^{1-\beta_{2} / \gamma}(\vartheta(\eta))\right]_{\eta=t_{2}}^{t} \leq \frac{1}{\vartheta_{0}\left(\beta_{2} / \gamma-1\right)} w^{1-\beta_{2} / \gamma}\left(\vartheta\left(t_{2}\right)\right),
$$

then (20) becomes

$$
\int_{t_{2}}^{\infty}\left[\frac{1}{r(\eta)} \int_{\eta}^{\infty} q(\zeta) P^{\beta}(\vartheta(\zeta)) d \zeta\right]^{1 / \gamma} d \eta<\infty
$$

which is a contradiction to (A9). This contradiction implies that the solution $x$ cannot be eventually positive. The case where $x$ is eventually negative is very similar, and we omit it here.

We finalize the paper by presenting some examples to show effectiveness and feasibility of the main results and Remark 3.3.

Example 3.1 Consider the differential equation

$$
\left(t\left(\left(x(t)+\frac{1}{t} x^{\frac{1}{3}}\left(\frac{t}{2}\right)+\frac{1}{t^{2}} x^{\frac{1}{5}}\left(\frac{t}{3}\right)\right)^{\prime}\right)^{3}\right)^{\prime}+t^{6} x^{3}\left(\frac{t}{2}\right)=0 \quad \text { for } t \geq 4
$$


where $r(t): \equiv y, q(t): \equiv t^{6}, \vartheta(t): \equiv \frac{t}{2}, \beta=\gamma=3, p_{i}(t): \equiv \frac{1}{t^{i}}, \alpha_{i}: \equiv \frac{1}{2 i+1}$, and $\varsigma_{i}(t): \equiv \frac{t}{i+1}$ for $i=1,2, \ldots, m$, and $t \geq 4$. All the assumptions of Theorem 3.1 can be verified with the index $i=1,2$ and $\rho(t)=\frac{1}{t}$. Hence, due to Theorem 3.1 every solution of (21) is oscillatory.

Example 3.2 Consider the differential equation

$$
\left(t\left(\left(x(t)+\frac{1}{t} x^{\frac{1}{3}}\left(\frac{t}{3}\right)+\frac{1}{t^{2}} x^{\frac{1}{5}}\left(\frac{t}{4}\right)\right)^{\prime}\right)^{5}\right)^{\prime}+t^{\frac{6}{5}} x\left(\frac{t}{2}\right)=0 \quad \text { for } t \geq 4
$$

where $r(t): \equiv t, q(t): \equiv t^{\frac{6}{5}}, \vartheta(t): \equiv \frac{t}{2}, \beta=1<\gamma=5, p_{i}(t): \equiv \frac{1}{t^{i}}, \alpha_{i}: \equiv \frac{1}{2 i+1}$, and $\varsigma_{i}(t): \equiv \frac{t}{i+2}$ for $i=1,2, \ldots, m$ and $t \geq 2$. All the assumptions of Theorem 3.4 (or Theorem 3.1) can be verified with the index $i=1,2$ and $\rho(t)=\frac{1}{t}$. Hence, due to Theorem 3.4 (or Theorem 3.1) every solution of (22) is oscillatory.

Example 3.3 Consider the differential equation

$$
\left((t+1)\left(x(t)+\frac{1}{t^{2}} x^{\frac{1}{3}}\left(\frac{t}{2}\right)+\frac{1}{t^{4}} x^{\frac{3}{5}}\left(\frac{t}{3}\right)\right)^{\prime}\right)^{\prime}+t^{12} x^{3}\left(\frac{t}{2}\right)=0 \quad \text { for } t \geq 2
$$

where $r(t): \equiv t+1, q(t): \equiv t^{12}, \vartheta(t): \equiv \frac{t}{2}, \vartheta^{\prime}(t)>\frac{1}{3}=\vartheta_{0}, \beta=3>\gamma=1, p_{i}(t): \equiv \frac{1}{t^{2 i}}, \alpha_{i}: \equiv \frac{2 i-1}{2 i+1}$, and $\varsigma_{i}(t): \equiv \frac{t}{i+1}$ for $i=1,2, \ldots, m$ and $t \geq 2$. All the assumptions of Theorem 3.5 (or Theorem 3.1) can be verified with the index $i=1,2$ and $\rho(t)=\frac{1}{t^{2}}$. Hence, due to Theorem 3.4 (or Theorem 3.1), every solution of (23) is oscillatory.

\section{Conclusion}

In this work, we have undertaken the problem by taking a second-order nonlinear neutral differential equation with sublinear neutral terms and established the sufficient conditions for oscillation of (1). However, we failed to establish the necessary and sufficient conditions for oscillation of all solutions of (1) by using the method adopted in the current paper. It seems that some other method may be required to establish the necessary and sufficient conditions for oscillation.

\section{Acknowledgements}

The authors express their debt of gratitude to the editors and the anonymous referee for accurate reading of the manuscript and beneficial comments, which improved the content of this paper.

Funding

The authors received no direct funding for this work.

Availability of data and materials

Not applicable.

Competing interests

The authors declare that they have no competing interests.

Authors' contributions

The authors declare that they have read and approved the final manuscript.

Authors' information

Not applicable.

\section{Author details}

'Department of Mathematics, JIS College of Engineering, Kalyani, 741235, India. ${ }^{2}$ Department of Mathematics, Faculty of Science, Hadhramout University, Hadhramout 50512, Yemen. ${ }^{3}$ Department of Mathematics, Faculty of Education, Seiyun University, Hadhramout 50512, Yemen. 


\section{Publisher's Note}

Springer Nature remains neutral with regard to jurisdictional claims in published maps and institutional affiliations.

Received: 1 July 2020 Accepted: 4 November 2020 Published online: 17 November 2020

\section{References}

1. Agarwal, R.P., Bohner, M., Li, T., Zhang, C.: Oscillation of second-order differential equations with a sublinear neutral term. Carpath. J. Math. 30, 1-6 (2014)

2. Agarwal, R.P., Bohner, M., Li, T., Zhang, C.: Oscillation of second-order Emden-Fowler neutral delay differential equations. Ann. Mat. Pura Appl. 193(4), 1861-1875 (2014)

3. Agarwal, R.P., Bohner, M., Li, T., Zhang, C.: Even-order half-linear advanced differential equations: improved criteria in oscillatory and asymptotic properties. Appl. Math. Comput. 266, 481-490 (2015)

4. Agarwal, R.P., Zhang, C., Li, T.: Some remarks on oscillation of second order neutral differential equations. Appl. Math. Comput. 274, 178-181 (2016)

5. Ataeea, P., Hahn, J.O., Dumont, G.A., Noubari, H.A., Boyce, W.T.: A model-based approach to stability analysis of autonomic-cardiac regulation. Comput. Biol. Med. 61(1), 119-126 (2015)

6. Baculikova, B., Dzurina, J.: Oscillation theorems for second-order neutral differential equations. Comput. Math. Appl. 61, 94-99 (2011)

7. Baculikova, B., Dzurina, J.: Oscillation theorems for second-order nonlinear neutral differential equations. Comput. Math. Appl. 62, 4472-4478 (2011)

8. Baculikova, B., Li, T., Dzurina, J.: Oscillation theorems for second order neutral differential equations. Electron. J. Qual. Theory Differ. Equ. 2011, 74 (2011)

9. Bohner, M., Grace, S.R., Jadlovska, I.: Oscillation criteria for second-order neutral delay differential equations. Electron. J. Qual. Theory Differ. Equ. 2017, 60 (2017)

10. Bohner, M., Hassan, T.S., Li, T.: Fite-Hille-Wintner-type oscillation criteria for second-order half-linear dynamic equations with deviating arguments. Indag. Math. 29(2), 548-560 (2018)

11. Brands, J.J.M.S.: Oscillation theorems for second-order functional-differential equations. J. Math. Anal. Appl. 63(1), 54-64 (1978)

12. Chatzarakis, G.E., Dzurina, J., Jadlovska, l.: New oscillation criteria for second-order half-linear advanced differential equations. Appl. Math. Comput. 347, 404-416 (2019)

13. Chatzarakis, G.E., Elabbasy, E.M., Bazighifan, O.: An oscillation criterion in 4th-order neutral differential equations with a continuously distributed delay. Adv. Differ. Equ. 2019, 336 (2019)

14. Chatzarakis, G.E., Grace, S.R., Jadlovská, I., Li, T., Tunç, E.: Oscillation criteria for third-order Emden-Fowler differential equations with unbounded neutral coefficients. Complexity 2019, Article ID 5691758 (2019)

15. Chatzarakis, G.E., Jadlovska, I.: Improved oscillation results for second-order half-linear delay differential equations. Hacet. J. Math. Stat. 48(1), 170-179 (2019)

16. Džurina, J.: Oscillation theorems for second-order advanced neutral differential equations. Tatra Mt. Math. Publ. 48, 61-71 (2011)

17. Džurina, J., Grace, S.R., Jadlovska, I., Li, T.: Oscillation criteria for second-order Emden-Fowler delay differential equations with a sublinear neutral term. Math. Nachr. 293(5), 910-922 (2020)

18. Fisnarova, S., Marik, R.: Oscillation of neutral second-order half-linear differential equations without commutativity in delays. Math. Slovaca 67(3), 701-718 (2017)

19. Grace, S.R., Džurina, J., Jadlovska, I., Li, T.: An improved approach for studying oscillation of second-order neutral delay differential equations. J. Inequal. Appl. 2018, 193 (2018)

20. Hale, J.K.: Theory of Functional Differential Equations, 2nd edn. Applied Mathematical Sciences, vol. 3. Springer, New York (1977)

21. Karpuz, B., Santra, S.S.: Oscillation theorems for second-order nonlinear delay differential equations of neutral type. Hacet. J. Math. Stat. 48(3), 633-643 (2019)

22. Li, H., Zhao, Y., Han, Z.: New oscillation criterion for Emden-Fowler type nonlinear neutral delay differential equations. J. Appl. Math. Comput. 60(1-2), 191-200 (2019)

23. Li, T., Pintus, N., Viglialoro, G.: Properties of solutions to porous medium problems with different sources and boundary conditions. Z. Angew. Math. Phys. 70(3), Art. 86 (2019)

24. Li, T., Rogovchenko, Y.V.: Oscillation of second-order neutral differential equations. Math. Nachr. 288, 1150-1162 (2015)

25. Li, T., Rogovchenko, Y.V.: Oscillation criteria for second-order superlinear Emden-Fowler neutral differential equations Monatshefte Math. 184, 489-500 (2017)

26. Li, T., Rogovchenko, Y.V.: On the asymptotic behavior of solutions to a class of third-order nonlinear neutral differential equations. Appl. Math. Lett. 105, Art. 106293 (2020)

27. Ottesen, J.T.: Modelling of the baroreflex-feedback mechanism with time-delay. J. Math. Biol. 36(1), 41-63 (1997)

28. Pinelas, S., Santra, S.S.: Necessary and sufficient condition for oscillation of nonlinear neutral first-order differential equations with several delays. J. Fixed Point Theory Appl. 20, 27 (2018)

29. Pinelas, S., Santra, S.S.: Necessary and sufficient conditions for oscillation of nonlinear first order forced differential equations with several delays of neutral type. Analysis 39(3), 97-105 (2019)

30. Santra, S.S.: Existence of positive solution and new oscillation criteria for nonlinear first-order neutral delay differential equations. Differ. Equ. Appl. 8(1), 33-51 (2016)

31. Santra, S.S.: Oscillation analysis for nonlinear neutral differential equations of second-order with several delays. Mathematica 59(82)(1-2), 111-123 (2017)

32. Santra, S.S.: Oscillation analysis for nonlinear neutral differential equations of second-order with several delays and forcing term. Mathematica 61(84)(1), 63-78 (2019)

33. Santra, S.S.: Necessary and sufficient condition for oscillatory and asymptotic behavior of second-order functional differential equations. Kragujev. J. Math. 44(3), 459-473 (2020)

34. Santra, S.S.: Necessary and sufficient conditions for oscillatory and asymptotic behavior of solutions to second-order nonlinear neutral differential equations with several delays. Tatra Mt. Math. Publ. 75, 121-134 (2020) 
35. Santra, S.S., Dix, J.G.: Necessary and sufficient conditions for the oscillation of solutions to a second-order neutral differential equation with impulses. Nonlinear Stud. 27(2), 375-387 (2020)

36. Tripathy, A.K., Panda, B., Sethi, A.K.: On oscillatory nonlinear second-order neutral delay differential equations. Differ Equ. Appl. 8(2), 247-258 (2016)

37. Wong, J.S.W.: Necessary and sufficient conditions for oscillation of second-order neutral differential equations. J. Math. Anal. Appl. 252(1), 342-352 (2000)

Submit your manuscript to a SpringerOpen ${ }^{\circ}$ journal and benefit from:

- Convenient online submission

- Rigorous peer review

- Open access: articles freely available online

- High visibility within the field

- Retaining the copyright to your article

Submit your next manuscript at $\boldsymbol{~ s p r i n g e r o p e n . c o m ~}$ 